\title{
NORMALIZING CONSTANTS FOR A STATISTIC BASED ON LOGARITHMS OF DISJOINT $m$-SPACINGS
}

Abstract. The paper is concerned with the asymptotic normality of a certain statistic based on the logarithms of disjoint $m$-spacings. The exact and asymptotic mean and variance are computed in the case of uniform distribution on the interval $[0,1]$. This result is generalized to the case when the sample is drawn from a distribution with positive step density on $[0,1]$.

1. Introduction. Let $X_{1}, \ldots, X_{N}$ be a sample from the uniform distribution on the interval $[0,1]$. Let us denote by $X_{(1)}, \ldots, X_{(N)}$ the order statistics derived from this sample and define $X_{(0)}=0, X_{(N+1)}=1$. Let $Y_{0}, Y_{1}, \ldots, Y_{N}$ be i.i.d. exponential random variables with unit mean. It is known that

$$
X_{(i)} \stackrel{d}{=} \exp \left(-\frac{Y_{i}}{i}-\ldots-\frac{Y_{N}}{N}\right), \quad i=1, \ldots, N .
$$

We will use the notation

$$
X_{k: l}=\exp \left(-\sum_{i=k}^{l} \frac{Y_{i}}{i}\right), \quad 1 \leq k \leq l \leq N .
$$

We also have

$$
X_{(i)} \stackrel{d}{=} \frac{Y_{1}+\ldots+Y_{i}}{Y_{0}+\ldots+Y_{N}} .
$$

We define the $m$-spacings from the sample $X_{1}, \ldots, X_{N}$ as the differences $D_{n, N}^{(m)}=X_{(n+m)}-X_{(n)}, n=0, \ldots, N+1-m, m \geq 1$. Let us introduce the

1991 Mathematics Subject Classification: 62E15, 62E20, 62G10, 62G30.

Key words and phrases: higher-order spacings; asymptotic normality; step density function.

Research supported by KBN (Committee for Scientific Research, Poland) Grant 2 10339101. 
following notation:

$$
G_{n}^{(m)}=D_{n, N}^{(m)} \log D_{n, N}^{(m)}
$$

We are interested in the statistic

$$
G_{N}=\sum_{0 \leq i m \leq N+1-m} G_{i m}^{(m)}
$$

based on disjoint $m$-spacings. It can be proved as in Cressie (1976) or deduced from Del Pino (1979) that $G_{N}$ is asymptotically normal. The general results of Del Pino (1979) give the asymptotic means and variances of statistics based on disjoint $m$-spacings in the form of means and variances of some other random variables. In any particular case these quantities have to be computed separately to obtain the values of normalizing constants. This method was used by Jammalamadaka \& Tiwari (1986). In this paper we will find the exact values of $\mathrm{E}\left(G_{N}\right)$ and $\operatorname{Var}\left(G_{N}\right)$ in a closed and simple form. We will also establish the asymptotic normality of $G_{N}$ for alternatives with step densities.

\section{Main results}

Theorem 1. Let $k \geq 1$ and $N+1=\mathrm{km}$. Then the random variable $G_{N}$ has the mean and variance given by

$$
\begin{aligned}
\mathrm{E}\left(G_{N}\right) & =-\sum_{i=m+1}^{N+1} \frac{1}{i}, \\
\operatorname{Var}\left(G_{N}\right) & =\frac{1}{N+2}[F(m+1)-F(N+2)],
\end{aligned}
$$

where

$$
F(n)=n \sum_{i=n}^{\infty} \frac{1}{i^{2}}
$$

The method of the proof will be similar to that of Cressie (1976). To prove the main result we will need some lemmas.

LEMMA 1 . For $\alpha>-1$ and $n \geq 1$ we have

$$
\mathrm{E}\left(X_{n: N}^{\alpha}\right)=\prod_{i=n}^{N} \frac{i}{i+\alpha}
$$

(b) $\mathrm{E}\left(X_{n: N}^{\alpha} \log X_{n: N}\right)=-\prod_{i=n}^{N} \frac{i}{i+\alpha} \sum_{j=n}^{N} \frac{1}{j+\alpha}$,

(c) $\mathrm{E}\left(X_{n: N}^{\alpha} \log ^{2} X_{n: N}\right)=\prod_{i=n}^{N} \frac{i}{i+\alpha}\left[\sum_{j=n}^{N} \frac{1}{(j+\alpha)^{2}}+\left(\sum_{j=n}^{N} \frac{1}{j+\alpha}\right)^{2}\right]$. 
Proof. The proof is straightforward using the definition of $X_{n: N}$ and integrating with respect to the density of the exponential distribution.

Lemma 2. For $n, m \geq 1$ we have

$$
\mathrm{E}\left(\log \left(1-X_{n: n+m-1}\right)\right)=-\sum_{k=1}^{\infty} \frac{1}{k} \prod_{i=n}^{n+m-1} \frac{i}{i+k}=-\sum_{i=m}^{n+m-1} \frac{1}{i} .
$$

Proof. Expanding the logarithm into a power series we get

$$
\begin{aligned}
\mathrm{E}\left(\log \left(1-X_{n: n+m-1}\right)\right) & =\mathrm{E}\left(-\sum_{k=1}^{\infty} \frac{1}{k} X_{n: n+m-1}^{k}\right) \\
& =-\sum_{k=1}^{\infty} \frac{1}{k} \mathrm{E}\left(X_{n: n+m-1}^{k}\right)=-\sum_{k=1}^{\infty} \frac{1}{k} \prod_{i=n}^{n+m-1} \frac{i}{i+k} .
\end{aligned}
$$

On the other hand, $X_{n: n+m-1}$ has the same distribution as the $n$th order statistic from a sample of size $n+m-1$. Applying the transformation $x \rightarrow 1-x$ we get $1-X_{n: n+m-1} \stackrel{d}{=} X_{m: n+m-1}$ and hence

$$
\mathrm{E}\left(\log \left(1-X_{n: n+m-1}\right)\right)=\mathrm{E}\left(\log X_{m: n+m-1}\right)=-\sum_{i=m}^{n+m-1} \frac{1}{i} .
$$

Lemma 3. For $n, m \geq 1$ we have

$$
\sum_{k=1}^{\infty} \prod_{i=n}^{n+m} \frac{i}{i+k}=\frac{n}{m}
$$

Pro of. Using Lemma 1 and the transformation $x \rightarrow 1-x$ as in the proof of Lemma 2, we get

$$
\begin{aligned}
\sum_{k=1}^{\infty} \prod_{i=n}^{n+m} \frac{i}{i+k} & =\sum_{k=1}^{\infty} \mathrm{E}\left(X_{n: n+m}^{k}\right)=\mathrm{E}\left(\sum_{k=1}^{\infty} X_{n: n+m}^{k}\right)=\mathrm{E}\left(\frac{X_{n: n+m}}{1-X_{n: n+m}}\right) \\
& =\mathrm{E}\left(\frac{1-X_{m+1: n+m}}{X_{m+1: n+m}}\right)=\mathrm{E}\left(\frac{1}{X_{m+1: n+m}}\right)-1 \\
& =\mathrm{E}\left(\exp \left(\sum_{i=m+1}^{n+m} \frac{Y_{i}}{i}\right)\right)-1=\prod_{i=m+1}^{n+m} \frac{i}{i-1}-1=\frac{n}{m} .
\end{aligned}
$$

Lemma 4. For $n, m \geq 1$ we have

$$
\sum_{k=1}^{\infty} \prod_{i=n}^{n+m-1} \frac{i}{i+k} \sum_{i=n}^{n+m-1} \frac{1}{i(i+k)}=\sum_{k=n+m}^{\infty} \frac{1}{k^{2}}
$$

Proof. This can be proved by induction. See Cressie (1976).

Proof of Theorem 1. We calculate $\mathrm{E}\left(G_{N}\right)$ first. It follows from (3) that all $m$-spacings have the same distribution. Hence from Lemma $1(\mathrm{~b})$ we 
get

$$
\begin{aligned}
\mathrm{E}\left(G_{n}^{(m)}\right) & =\mathrm{E}\left(G_{0}^{(m)}\right)=\mathrm{E}\left(X_{(m)} \log X_{(m)}\right) \\
& =\mathrm{E}\left(X_{m: N} \log X_{m: N}\right)=-\frac{m}{N+1} \sum_{i=m}^{N} \frac{1}{i+1} .
\end{aligned}
$$

Thus

$$
\begin{aligned}
\mathrm{E}\left(G_{N}\right) & =\mathrm{E}\left(\sum_{i=0}^{k-1} G_{i m}^{(m)}\right)=k \mathrm{E}\left(G_{0}^{(m)}\right) \\
& =\frac{N+1}{m} \cdot \frac{-m}{N+1} \sum_{i=m}^{N} \frac{1}{i+1}=-\sum_{i=m}^{N} \frac{1}{i+1} .
\end{aligned}
$$

The variance of $G_{N}$ is more complicated to obtain. We have

$$
\operatorname{Var}\left(G_{N}\right)=k \operatorname{Var}\left(G_{0}^{(m)}\right)+k(k-1) \operatorname{Cov}\left(G_{0}^{\left.(m), G_{m}^{(m)}\right)} .\right.
$$

From Lemma 1 we get

$$
\begin{aligned}
\operatorname{Var}\left(G_{0}^{(m)}\right)= & \mathrm{E}\left(X_{m: N}^{2} \log ^{2} X_{m: N}\right)-\mathrm{E}^{2}\left(X_{m: N} \log X_{m: N}\right) \\
= & \frac{m(m+1)}{(N+1)(N+2)}\left[\sum_{i=m+2}^{N+2} \frac{1}{i^{2}}+\left(\sum_{i=m+2}^{N+2} \frac{1}{i}\right)^{2}\right] \\
& -\frac{m^{2}}{(N+1)^{2}}\left(\sum_{i=m+1}^{N+1} \frac{1}{i}\right)^{2} .
\end{aligned}
$$

To calculate $\operatorname{Cov}\left(G_{0}^{(m)}, G_{n}^{(m)}\right), n \geq m$, let us note that

$$
\begin{aligned}
\log \left(X_{n+m: N}-X_{n: N}\right) & =\log X_{n+m: N}+\log \left(1-\frac{X_{n: N}}{X_{n+m: N}}\right) \\
& =\log X_{n+m: N}+\log \left(1-X_{n: n+m-1}\right) .
\end{aligned}
$$

Hence

$$
\begin{aligned}
\mathrm{E}\left(G_{0}^{(m)} G_{n}^{(m)}\right)= & \mathrm{E}\left(X_{m: N} \log X_{m: N}\left(X_{n+m: N}-X_{n: N}\right) \log X_{n+m: N}\right) \\
& +\mathrm{E}\left(X_{m: N} \log X_{m: N}\left(X_{n+m: N}-X_{n: N}\right) \log \left(1-X_{n: m+n-1}\right)\right) .
\end{aligned}
$$

The expected values above can be calculated using the identities

$$
X_{n: N}=X_{n: n+m-1} X_{n+m: N}, \quad X_{m: N}=X_{m: n-1} X_{n: N}
$$

and the fact that $X_{m: n-1}, X_{n: n+m-1}, X_{n+m: N}$ are independent. After elementary but lengthy calculations we get

(9) $\quad \mathrm{E}\left(X_{m: N} \log X_{m: N}\left(X_{n+m: N}-X_{n: N}\right) \log X_{n+m: N}\right)$

$$
=\frac{m^{2}}{(N+1)(N+2)}\left(\sum_{i=m+n+2}^{N+2} \frac{1}{i} \sum_{i=m+1}^{N+2} \frac{1}{i}+\sum_{i=m+n+2}^{N+2} \frac{1}{i^{2}}\right) .
$$


Similarly, using the expansion $\log \left(1-X_{n: m+n-1}\right)=-\sum_{k=1}^{\infty} k^{-1} X_{n: m+n-1}^{k}$ and Lemmas $1-4$ we obtain

(10) $\quad \mathrm{E}\left(X_{m: N} \log X_{m: N}\left(X_{n+m: N}-X_{n: N}\right) \log \left(1-X_{n: n+m-1}\right)\right)$

$$
=\frac{m^{2}}{(N+1)(N+2)}\left(\sum_{i=m+1}^{m+n+1} \frac{1}{i} \sum_{i=m+1}^{N+2} \frac{1}{i}-\sum_{i=m+n+2}^{\infty} \frac{1}{i^{2}}\right) .
$$

Combining (9) and (10) we have for $n \geq m$,

$$
\mathrm{E}\left(G_{0}^{(m)} G_{n}^{(m)}\right)=\frac{m^{2}}{(N+1)(N+2)}\left[\left(\sum_{i=m+1}^{N+2} \frac{1}{i}\right)^{2}-\sum_{i=N+3}^{\infty} \frac{1}{i^{2}}\right]
$$

and

$$
\begin{aligned}
\operatorname{Cov}\left(G_{0}^{(m)}, G_{n}^{(m)}\right)= & \mathrm{E}\left(G_{0}^{(m)} G_{n}^{(m)}\right)-\mathrm{E}\left(G_{0}^{(m)}\right) \mathrm{E}\left(G_{n}^{(m)}\right) \\
= & \frac{m^{2}}{N+1}\left[\frac{1}{N+2}\left(\sum_{i=m+1}^{N+2} \frac{1}{i}\right)^{2}\right. \\
& \left.-\frac{1}{N+1}\left(\sum_{i=m+1}^{N+1} \frac{1}{i}\right)^{2}-\frac{1}{N+2} \sum_{i=N+3}^{\infty} \frac{1}{i^{2}}\right] .
\end{aligned}
$$

Now we can calculate $\operatorname{Var}\left(G_{N}\right)$ :

$$
\begin{aligned}
\operatorname{Var}\left(G_{N}\right)= & k \operatorname{Var}\left(G_{0}^{(m)}\right)+k(k-1) \operatorname{Cov}\left(G_{0}^{(m)}, G_{m}^{(m)}\right) \\
= & \frac{m+1}{N+2}\left[\sum_{i=m+2}^{N+2} \frac{1}{i^{2}}+\left(\sum_{i=m+2}^{N+2} \frac{1}{i}\right)^{2}\right]-\frac{m}{N+1}\left(\sum_{i=m+1}^{N+1} \frac{1}{i}\right)^{2} \\
& +(k-1) m\left[\frac{1}{N+2}\left(\sum_{i=m+1}^{N+2} \frac{1}{i}\right)^{2}\right. \\
& \left.-\frac{1}{N+1}\left(\sum_{i=m+1}^{N+1} \frac{1}{i}\right)^{2}-\frac{1}{N+2} \sum_{i=N+3}^{\infty} \frac{1}{i^{2}}\right] \\
= & \frac{m+1}{N+2} \sum_{i=m+2}^{N+2} \frac{1}{i^{2}}-\frac{(k-1) m}{N+2} \sum_{i=N+3}^{\infty} \frac{1}{i^{2}}+\left(\sum_{i=m+1}^{N+2} \frac{1}{i}\right)^{2} \\
& -\left(\sum_{i=m+1}^{N+1} \frac{1}{i}\right)^{2}+\frac{m+1}{N+2}\left[\left(\sum_{i=m+2}^{N+2} \frac{1}{i}\right)^{2}-\left(\sum_{i=m+1}^{N+2} \frac{1}{i}\right)^{2}\right] \\
= & \frac{1}{N+2}\left[(m+1) \sum_{i=m+1}^{\infty} \frac{1}{i^{2}}-(N+2) \sum_{i=N+2}^{\infty} \frac{1}{i^{2}}\right] .
\end{aligned}
$$

In the next lemma we give the expected value and the variance of $G_{N}$ in the case when $N+1$ is not divisible by $m$. 
Lemma 5. Assume that $N+1$ is not divisible by $m$ and let $n<N$ be the largest integer such that $n+1$ is divisible by $m$, i.e. $n+1=k m$. Then

(12) $\quad \operatorname{Var}\left(G_{N}\right)=\frac{(n+1)(n+2)}{(N+1)(N+2)}$

$$
\begin{aligned}
& \times\left[\sum_{i=n+3}^{N+2} \frac{1}{i^{2}}+\left(\sum_{i=n+3}^{N+2} \frac{1}{i}+\sum_{i=m+1}^{n+1} \frac{1}{i}\right)^{2}+\operatorname{Var}\left(G_{n}\right)\right] \\
& -\frac{(n+1)^{2}}{(N+1)^{2}}\left(\sum_{i=m+1}^{N+1} \frac{1}{i}\right)^{2}
\end{aligned}
$$

where $\operatorname{Var}\left(G_{n}\right)$ is given by $(7)$.

Pro of. We have $G_{N}=\sum_{i=0}^{k-1} D_{i m, N}^{(m)} \log D_{i m, N}^{(m)}$ and

$$
\begin{aligned}
D_{i m, N}^{(m)} & =\left(X_{i m+m: N}-X_{i m: N}\right) \\
& =X_{n+1: N}\left(X_{i m+m: n}-X_{i m: n}\right)=X_{n+1: N} D_{i m, n}^{(m)} .
\end{aligned}
$$

In the equation above $X_{n+1: N}$ is independent of $D_{i m, n}^{(m)}$, thus

$$
\begin{aligned}
G_{N} & =\sum_{i=0}^{k-1} X_{n+1: N} D_{i m, n}^{(m)} \log \left(X_{n+1: N} D_{i m, n}^{(m)}\right) \\
& =\sum_{i=0}^{k-1} X_{n+1: N} \log X_{n+1: N} D_{i m, n}^{(m)}+\sum_{i=0}^{k-1} X_{n+1: N} D_{i m, n}^{(m)} \log D_{i m, n}^{(m)} \\
& =X_{n+1: N} \log X_{n+1: N}+X_{n+1: N} G_{n}
\end{aligned}
$$

and $X_{n+1: N}$ is independent of $G_{n}$. Using the last identity and applying Lemma 1 we get easily the statement of Lemma 5 .

Theorem 2. $\mathrm{E}\left(G_{N}\right)$ and $\operatorname{Var}\left(G_{N}\right)$ given by (6), (11) and (7), (12) respectively are asymptotically equivalent to

$$
e_{N}=\sum_{i=1}^{m} \frac{1}{i}-\log (N+2)-\gamma
$$

and

$$
\sigma_{N}^{2}=\frac{1}{N+2}[F(m+1)-1],
$$

where $\gamma=0.577 \ldots$ is Euler's constant. 
Proof. We want to show that $\operatorname{Var}\left(G_{N}\right) / \sigma_{N}^{2} \rightarrow 1$ and $\left(\mathrm{E}\left(G_{N}\right)-e_{N}\right) / \sigma_{N}$ $\rightarrow 0$. Since $F(n) \rightarrow 1$ it is easy to show that (7) and (12) are equivalent to $\sigma_{N}^{2}$. It remains to prove that $\sqrt{N+2}\left(\mathrm{E}\left(G_{N}\right)-e_{N}\right) \rightarrow 0$. We can replace (11) by (6) because

$$
\sqrt{N+2}\left|-\frac{n+1}{N+1} \sum_{i=m+1}^{N+1} \frac{1}{i}+\sum_{i=m+1}^{N+1} \frac{1}{i}\right| \leq \frac{m \sqrt{N+2}}{N+1} \sum_{i=m+1}^{N+1} \frac{1}{i} \rightarrow 0 .
$$

Let us define $\gamma_{N}=\sum_{i=1}^{N} i^{-1}-\log (N+1)$. Then

$$
\gamma_{N}=\sum_{i=1}^{N}\left[\frac{1}{i}-\log \left(\frac{i+1}{i}\right)\right]
$$

and $\gamma_{N} \rightarrow \gamma$. Applying the inequalities

$$
0<\frac{1}{i}-\log \left(\frac{i+1}{i}\right)<\frac{1}{i}-\frac{1}{i+1}<\frac{1}{i^{2}}
$$

we get

$$
\begin{aligned}
\sqrt{N+2} & \left(-\sum_{i=m+1}^{N+1} \frac{1}{i}-e_{N}\right) \\
& =\sqrt{N+2}\left(\gamma-\gamma_{N+1}\right)=\sqrt{N+2} \sum_{i=N+2}^{\infty}\left(\frac{1}{i}-\log \left(\frac{i+1}{i}\right)\right) \\
& \leq \sqrt{N+2} \sum_{i=N+2}^{\infty} \frac{1}{i^{2}} \leq \sum_{i=N+2}^{\infty} \frac{1}{i^{3 / 2}} \rightarrow 0 .
\end{aligned}
$$

It is easy to see that the expresions for $e_{N}$ and $\sigma_{N}^{2}$ given by (13) and (14) agree with the results of Gebert and Kale (1969) for $m=1$ and Jammalamadaka \& Tiwari (1986) for $m \geq 1$.

3. The case of step densities. In this section we will find the asymptotic distribution of $G_{n}$ in the case when the underlying distribution has a positive step density on $[0,1]$. For $m=1$ it was found by Czekała (1993).

Let $\underline{X}=\left(X_{1}, X_{2}, \ldots, X_{n}, \ldots\right)$ be a sequence of random variables which are used to form $G_{n} . G_{n}$ is a function of $n$ and $\underline{X}$ which we can write as $G_{n}=\phi(n, \underline{X})$. To simplify the notation we assume that $\phi(0, \underline{X})=0$. Now let $k \geq 1$ be a fixed integer and let $0=x_{0}<x_{1}<\ldots<x_{k}=1$ be fixed real numbers. We can define subintervals of $[0,1]$ as follows:

$$
I_{1}=\left[x_{0}, x_{1}\right), \ldots, I_{k-1}=\left[x_{k-2}, x_{k-1}\right), \quad I_{k}=\left[x_{k-1}, x_{k}\right] .
$$

The lengths of the intervals $I_{i}$ will be denoted by $d_{i}$. Let $f_{i}>0, i=1, \ldots, k$, be fixed numbers such that $\sum_{i=1}^{k} f_{i} d_{i}=1$. These numbers together with 
the intervals $I_{i}$ define a step density $f$ :

$$
f(x)=\sum_{i=1}^{k} f_{i} \mathbf{1}_{I_{i}}(x)
$$

Define $p_{i}=f_{i} d_{i}$. We have $\sum p_{i}=1$ and hence there exist numbers $0=x_{0}^{\prime}<$ $x_{1}^{\prime}<\ldots<x_{k}^{\prime}=1$ such that the intervals $I_{i}^{\prime}$, defined similarly to $I_{i}$, have lengths $p_{i}$. There also exists a vector of random elements $\left(\underline{U}, \underline{Y}^{1}, \ldots, \underline{Y}^{k}\right)$ such that:

(a) the coordinates of this vector are stochastically independent,

(b) $\underline{U}=\left(U_{1}, U_{2}, \ldots\right)$ is a sequence of independent random variables uniformly distributed on $[0,1]$,

(c) $\underline{Y}^{i}=\left(Y_{1}^{i}, Y_{2}^{i}, \ldots\right)$, for $i=1, \ldots, k$, are sequences of independent random variables with uniform distribution on $I_{i}$.

We can now define a sequence $\underline{Z}=\left(Z_{1}, Z_{2}, \ldots\right)$ of independent random variables with density $f$ :

$$
Z_{n}=\sum_{i=1}^{k} \mathbf{1}_{I_{i}^{\prime}}\left(U_{n}\right) Y_{n}^{i}, \quad n \geq 1 .
$$

Let us denote by $N_{i, n}$ the number of random variables $Z_{1}, \ldots, Z_{n}$ taking values in the interval $I_{i}$, that is,

$$
N_{i, n}=\sum_{j=1}^{n} \mathbf{1}_{I_{i}}\left(Z_{j}\right)
$$

It is easy to see that $N_{i, n}=\sum_{j=1}^{n} \mathbf{1}_{I_{i}^{\prime}}\left(U_{j}\right)$ and thus the sequence of vectors $\left(N_{1, n}, \ldots, N_{k, n}\right)$ is independent of $\left(\underline{Y}^{1}, \ldots, \underline{Y}^{k}\right)$. Using this we will prove in the next two lemmas that $\phi(n, \underline{Z})$ is asymptotically identically distributed as

$$
\sum_{i=1}^{k} \phi\left(N_{i, n}, \underline{Y}^{i}\right)
$$

In the sequel we will use the notation $\psi(x)=x \log x$.

LEMMA 6. If $\tau$ is an arbitrary random variable taking values in the set $\{1, \ldots, m\}$ then

$$
\sqrt{n} \psi\left(X_{(\tau)}\right) \stackrel{P}{\rightarrow} 0,
$$

where $X_{(\tau)}$ denotes the $\tau$-th order statistic from the sample of size $n$.

Proof. We have

$$
P\left(\sqrt{n}\left|\psi\left(X_{(\tau)}\right)\right| \geq \varepsilon\right) \leq \sum_{i=1}^{m} P\left(\sqrt{n}\left|\psi\left(X_{(i)}\right)\right| \geq \varepsilon\right),
$$


hence it is enough to prove that $\sqrt{n} \psi\left(X_{(i)}\right) \stackrel{P}{\rightarrow} 0$ for each fixed $i \geq 1$. It is known that $n X_{(i)}$ converges weakly to some random variable $X$ (with Erlang's distribution). Since $\psi$ is a continuous function we also have $\psi\left(n X_{(i)}\right)$ $\stackrel{d}{\rightarrow} \psi(X)$. It follows that

$$
\sqrt{n} \psi\left(X_{(i)}\right)=\frac{\psi\left(n X_{(i)}\right)}{\sqrt{n}}-\frac{\log n}{\sqrt{n}}\left(n X_{(i)}\right) \stackrel{P}{\rightarrow} 0 .
$$

LEMMA 7. The statistic $\phi(n, \underline{Z})$ has the same asymptotic distribution as the statistic (18).

P r o of. Let us consider the sequence $Z_{\alpha_{i}, 1}, Z_{\alpha_{i}, 2}, \ldots$ of those successive random variables $Z_{n}$ whose values belong to the interval $I_{i}$. The sequence $\alpha_{i, n}, n \geq 1$, is determined by the sequence $\underline{U}$, so it is independent of $\underline{Y}^{i}$. Since by (16), $Z_{\alpha_{i, n}}=Y_{\alpha_{i, n}}^{i}$, the sequence $Z_{\alpha_{i, n}}$ has the same probability distribution as $\underline{Y}^{i}$. It can be shown similarly that the joint distribution of the vector $\left(Z_{\alpha_{1, n}}, n \geq 1, \ldots, Z_{\alpha_{k, n}}, n \geq 1\right)$ is the same as that of $\left(\underline{Y}^{1}, \ldots, \underline{Y}^{k}\right)$. It follows that the sequence $X_{(1)}, \ldots, X_{(n)}$ has the same probability distribution as $Y_{(1)}^{1}, \ldots, Y_{\left(N_{1, n}\right)}^{1}, \ldots, Y_{(1)}^{k}, \ldots, Y_{\left(N_{k, n}\right)}^{k}$. To prove our lemma we need to exclude the $m$-spacings that span different intervals $I_{i}$. We have $N_{i, n} \stackrel{P .1}{\rightarrow} \infty$ so we can assume that each of the intervals $I_{i}$ contains at least one full $m$-spacing. Then the $m$-spacings that are fully contained in $I_{i}$ are built from the random variables

$$
Y_{\tau_{i}}^{i}, Y_{\tau_{i}+1}^{i}, \ldots, Y_{\tau_{i}+\eta_{i}}^{i}
$$

where $\tau_{i}=m+m\left[S_{i-1} / m\right]-S_{i-1}, \eta_{i}=m\left[S_{i} / m\right]-m\left[S_{i-1} / m\right]-m$ and $S_{i}=\sum_{j=1}^{i} N_{j, n}$. We have omitted the index $n$ in the definition of $\tau_{i}, \eta_{i}, S_{i}$ to simplify the notation. Let $D=Y_{\tau_{i+1}}^{i+1}-Y_{\tau_{i}+\eta_{i}}^{i}$ be the $m$-spacing containing $x_{i}$ which is the common endpoint of $I_{i}$ and $I_{i+1}$. We can write $D=D_{1}+D_{2}=$ $\left(x_{i}-Y_{\tau_{i}+\eta_{i}}^{i}\right)+\left(Y_{\tau i+1}^{i+1}-x_{i}\right)$. We have $\sqrt{n}|\psi(D)| \leq \sqrt{n}\left|\psi\left(D_{1}\right)\right|+\sqrt{n}\left|\psi\left(D_{2}\right)\right|$ and from Lemma 6 we obtain $\psi(D) \stackrel{P}{\rightarrow} 0$. We have proven that the $m$ spacings spanning different intervals $I_{i}$ are negligible.

Now $\tau_{i}$ and $\eta_{i}$ are independent of $\underline{Y}^{i}$ so the distribution of (19) will not change if we replace (19) by

$$
Y_{(0)}^{i}, Y_{(1)}^{i}, \ldots, Y_{\left(\eta_{i}\right)}^{i}
$$

It follows that $\phi(n, \underline{Z})$ has the same asymptotic distribution as $\sum_{i=1}^{k} \phi\left(\eta_{i}, \underline{Y}^{i}\right)$. It is easy to see that the latter statistic is asymptotically identically distributed as (18) because $0<N_{i, n}-\eta_{i}<2 m-1$ and $N_{i, n} \stackrel{P .1}{\rightarrow} \infty$.

We will need some lemmas to find the asymptotic distribution of (18). 
LemMA 8. Let $X$ and $X_{n}, n \geq 1$, be random variables such that $X_{n} \stackrel{P .1}{\rightarrow} 0$ and $\sqrt{n} X_{n} \stackrel{d}{\rightarrow} X$. Then

$$
\sqrt{n}\left|\log \left(1+X_{n}\right)-X_{n}\right| \stackrel{P}{\rightarrow} 0 .
$$

Proof. (21) follows easily from the fact that for $|x| \leq 1 / 2$ we have

$$
|\log (1+x)-x| \leq x^{2}
$$

Lemma 9. Let $X_{i, n}, i=1, \ldots, k, n \geq 0$, be the random variables defined as follows:

$$
X_{i, n}=\frac{1}{\sigma_{n}}\left(\phi\left(n, \underline{Y}^{i}\right)-d_{i}\left(\log d_{i}+e_{n}\right)\right),
$$

where $e_{n}$ and $\sigma_{n}$ are defined by (13) and (14). Then

$$
\begin{aligned}
\left(X_{1, N_{1, n}}, \ldots, X_{k, N_{k, n}}, \frac{N_{1, n}-n p_{1}}{\sqrt{n}}, \ldots, \frac{N_{k, n}-n p_{k}}{\sqrt{n}}\right) \\
\stackrel{d}{\rightarrow}\left(d_{1} X_{1}, \ldots, d_{k} X_{k}, W_{1}, \ldots, W_{k}\right),
\end{aligned}
$$

where the $X_{i}$ are independent and normally $N(0,1)$ distributed random variables, the vector $\left(W_{1}, \ldots, W_{k}\right)$ is independent of $\left(X_{1}, \ldots, X_{k}\right)$ and has the multivariate normal distribution $N(0, \Sigma)$, where $\Sigma=\left[\sigma_{i, j}\right]$ and

$$
\sigma_{i, j}= \begin{cases}-p_{i} p_{j} & \text { for } i \neq j \\ p_{i}-p_{i}^{2} & \text { for } i=j\end{cases}
$$

$i, j=1, \ldots, k$.

Proof. We will first show that

$$
\left(X_{1, n}, \ldots, X_{k, n}\right) \stackrel{d}{\rightarrow}\left(d_{1} X_{1}, \ldots, d_{k} X_{k}\right) .
$$

We have $\sigma_{n}^{-1}\left(\phi\left(n, \underline{Y}^{i} / d_{i}\right)-e_{n}\right) \stackrel{d}{\rightarrow} X_{i}$, where $e_{n}$ and $\sigma_{n}$ are given by (13) and (14) respectively. Transforming $\phi\left(n, \underline{Y}^{i} / d_{i}\right)$ into $\phi\left(n, \underline{Y}^{i}\right)$ we get $X_{i, n} \stackrel{d}{\rightarrow}$ $d_{i} X_{i}$. As $X_{i, n}$ are independent we also have (23). Now we show that

$$
\left(\frac{N_{1, n}-n p_{1}}{\sqrt{n}}, \ldots, \frac{N_{1, n}-n p_{1}}{\sqrt{n}}\right) \stackrel{d}{\rightarrow}\left(W_{1}, \ldots, W_{k}\right) .
$$

This follows from the central limit theorem because $N_{i, n}=\sum_{j=1}^{n} \mathbf{1}_{I_{i}^{\prime}}\left(U_{j}\right)$, $\mathrm{E}\left(\mathbf{1}_{I_{i}^{\prime}}\left(U_{1}\right)\right)=p_{i}$ and $\operatorname{Cov}\left(\mathbf{1}_{I_{i}^{\prime}}\left(U_{1}\right), \mathbf{1}_{I_{j}^{\prime}}\left(U_{1}\right)\right)=\sigma_{i, j}$. Since the sequence $N_{i, n}$ is independent of $\underline{Y}^{i},(23)$ and (24) together give (22).

LEMMA 10. We have

$$
\left(\sqrt{n} \log \left(\frac{N_{1, n}}{n p_{1}}\right), \ldots, \sqrt{n} \log \left(\frac{N_{k, n}}{n p_{k}}\right)\right) \stackrel{d}{\rightarrow}\left(\frac{1}{p_{1}} W_{1}, \ldots, \frac{1}{p_{k}} W_{k}\right) .
$$


Proof. Let $\alpha(x)=\log (1+x)-x$. Then

$$
\sqrt{n} \log \left(\frac{N_{i, n}}{n p_{i}}\right)=\frac{N_{i, n}-n p_{i}}{\sqrt{n} p_{i}}+\sqrt{n} \alpha\left(\frac{N_{i, n}-n p_{i}}{n p_{i}}\right) .
$$

From Lemma 8 it follows that $\sqrt{n} \alpha\left(\left(N_{i, n}-n p_{i}\right)\left(n p_{i}\right)^{-1}\right) \stackrel{P}{\rightarrow} 0$. The rest of the proof results from Lemma 9.

Now we can compute the asymptotic distribution of the statistic $G_{n}$ when the underlying distribution has density $f$ given by (15).

THEOREM 3. If the $X_{1}, X_{2}, \ldots, X_{n}, \ldots$ are i.i.d. random variables with the density $f$ given by (15) then

$$
\frac{G_{n}-e_{f, n}}{\sigma_{f, n}} \stackrel{d}{\rightarrow} N(0,1)
$$

where

$$
\begin{aligned}
e_{f, n} & =\mathrm{E}\left(\frac{1}{f} \log \left(\frac{1}{f}\right)\right)-\sum_{m+1}^{n+1} \frac{1}{i}, \\
\sigma_{f, n}^{2} & =\frac{1}{n+2}\left[F(m+1) \mathrm{E}\left(\frac{1}{f^{2}}\right)-1\right] .
\end{aligned}
$$

An asymptotically equivalent form of $e_{f, n}$ is

$$
e_{f, n}=\mathrm{E}\left(\frac{1}{f} \log \left(\frac{1}{f}\right)\right)-\log n+\sum_{i=1}^{m} \frac{1}{i}-\gamma .
$$

Proof. As proved in Lemma 7 we can replace $G_{n}$ by $\sum \phi\left(N_{i, n}, \underline{Y}^{i}\right)$. Set $C_{m}=F(m+1)-1$. From Lemmas 9 and 10 we get

$$
\begin{aligned}
\frac{1}{\sigma_{n}}\left[\sum_{i=1}^{k} \phi\left(N_{i, n}, \underline{Y}^{i}\right)-\sum_{i=1}^{k} d_{i} \log \left(\frac{d_{i}}{n p_{i}}\right)-\left(\sum_{i=1}^{m} \frac{1}{i}-\gamma\right)\right] \\
\stackrel{d}{\rightarrow} \sum_{i=1}^{k} \frac{d_{i}}{\sqrt{p_{i}}} X_{i}-\frac{1}{\sqrt{C_{m}}} \sum_{i=1}^{k} \frac{d_{i}}{p_{i}} W_{i},
\end{aligned}
$$

where $W_{i}$ and $X_{i}$ are defined in Lemma 9. It remains to compute the variance of the right side:

$$
\begin{aligned}
\operatorname{Var}\left(\sum_{i=1}^{k} \frac{d_{i}}{\sqrt{p_{i}}} X_{i}-\frac{1}{\sqrt{C_{m}}} \sum_{i=1}^{k} \frac{d_{i}}{p_{i}} W_{i}\right) & =\sum_{i=1}^{k} \frac{d_{i}^{2}}{p_{i}}+\frac{1}{C_{m}} \sum_{i=1}^{k} \sum_{j=1}^{k} \frac{d_{i} d_{j}}{p_{i} p_{j}} \sigma_{i j} \\
& =\sum_{i=1}^{k} \frac{d_{i}^{2}}{p_{i}}+\frac{1}{C_{m}}\left(\sum_{i=1}^{k} \frac{d_{i}^{2}}{p_{i}}-1\right) .
\end{aligned}
$$


From this we get

$$
\frac{\sum_{i=1}^{k} \phi\left(N_{i, n}, \underline{Y}^{i}\right)-\mathrm{E}\left(f^{-1} \log f^{-1}\right)+\log n-\sum_{i=1}^{m} i^{-1}+\gamma}{\sqrt{(n+2)^{-1}\left[F(m+1) \mathrm{E}\left(f^{-2}\right)-1\right]}} \stackrel{d}{\rightarrow} N(0,1) .
$$

Replacing $\log n+\gamma$ in the expression above by $\sum_{i=1}^{n+1} i^{-1}$, which is asymptotically equivalent as was shown in the proof of Theorem 2, we obtain (26).

\section{References}

N. Cressie (1976), On the logarithms of high-order spacings, Biometrika 63, 343-355.

F. Czekała (1993), Asymptotic distributions of statistics based on logarithms of spacings, Zastos. Mat. 21, 511-519.

G. E. Del Pino (1979) On the asymptotic distribution of $k$-spacings with applications to goodness of fit tests, Ann. Statist. 7, 1058-1065.

J. R. Gebert and B. K. Kale (1969), Goodness of fit tests based on discriminatory information, Statist. Hefte 3, 192-200.

S. R. J ammalamad aka and R. C. Tiw ari (1986), Efficiencies of some disjoint spacings tests relative to a $\chi^{2}$ test, in: M. L. Puri, J. Vilaplana and W. Wertz (eds.) New Perspectives in Theoretical and Applied Statistics, Wiley, New York, 311-318.

B. K. Kale (1969), Unified derivation of tests of goodness of fit based on spacings, Sankhyā Ser. A 31, 43-48.

Franciszek Czekała

Mathematical Institute

University of Wrocław

Pl. Grunwaldzki 2/4

50-384 Wrocław, Poland

E-mail: czekala@math.uni.wroc.pl 